\title{
Population biology of 225 serogroup 6 Streptococcus pneumoniae isolates collected in China
}

\author{
Wei Shi ${ }^{\dagger}$, Kaihu $\mathrm{YaO}^{\dagger}$, Mingming He, Sangjie Yu and Yonghong Yang ${ }^{*}$
}

\begin{abstract}
Background: In this study, we defined the population biology of serogroup 6 Streptococcus pneumoniae collected in China and their antibiotic resistance profiles.

Methods: The serotypes of 225 S. pneumoniae strains isolated between 1997 and 2011 were identified with the Quellung reaction and serotype-specific PCR. All isolated pneumococci were tested for their sensitivity to 11 kinds of antibiotics with the E-test method or disc diffusion. The sequence types (STs) were analyzed with multilocus sequence typing (MLST).

Results: The frequencies of serotypes and subtypes 6A, 6B-I, 6B-II, 6C, and 6D among the 225 isolates were 46.7\% (105/225), 19.6\% (44/225), 25.8\% (58/225), 6.2\% (14/225), and 1.8\% (4/225), respectively. Serotype 6E was not found in the serotype $6 \mathrm{~A}$ isolates, and neither serotype $6 \mathrm{~F}$ nor $6 \mathrm{G}$ was identified in any isolate. MLST analysis revealed 58 STs. The most common STs were ST982 (23.1\%), ST90 (14.7\%), ST4542 (7.6\%), and ST2912 (4.9\%). The rates of clonal complex 90 (CC90) and CC386 among the oral-penicillin-nonsusceptible isolates decreased over the years, whereas the rates of CC855 and CC3173 increased. The four CCs had similar penicillin MIC distributions, with a maximum MIC of $2 \mu \mathrm{g} / \mathrm{ml}$.
\end{abstract}

Conclusions: This study identified the serotypes/subtypes and CCS/STs of group 6 S. pneumoniae present in China. No salient antibiotic-resistant clones were isolated among the serogroup 6 S. pneumoniae.

Keywords: Streptococcus pneumoniae, Serotypes, Antibiotic resistance, Children, Epidemiology

\section{Background}

Streptococcus pneumoniae remains the leading cause of community-acquired respiratory infections, and is one of the most important human pathogens causing severe invasive infections [1]. So far, more than 90 pneumococcal serotypes, classified into 46 groups, have been identified. Serogroup 6 is one of the most common causes of invasive pneumococcal disease in unvaccinated populations. Serogroup 6 traditionally comprises two serotypes, 6A and $6 \mathrm{~B}$. Two new serotypes, $6 \mathrm{C}$ and $6 \mathrm{D}$, previously identified as serotype $6 \mathrm{~A}$ and $6 \mathrm{~B}$, respectively, by the Quellung reaction were identified as two distinct serotypes using a

\footnotetext{
*Correspondence: yyh628628@sina.com

${ }^{\dagger}$ Equal contributors

Key Laboratory of Major Diseases in Children and National Key Discipline of Pediatrics (Capital Medical University), Ministry of Education, Beijing Pediatric Research Institute, Beijing Children's Hospital Capital Medical University, Beijing, China
}

\section{Biomed Central}

multibead assay with monoclonal antibodies [2,3]. A factor antiserum, 6d, was then developed against these two new serotypes. Therefore, these two serotypes can now be identified with the traditional Quellung test $[4,5]$. Two subtypes of 6B, designated 6B-I and 6B-II, can be distinguished by polymerase chain reaction (PCR) [6]. Another study in Korea demonstrated that some serogroup 6 isolates encode distinctive cps (capsular) loci, differing from those of most other serogroup 6 isolates. These were designated the " $6 \mathrm{X}$ " group, and include both traditional serotype $6 \mathrm{~A}$ and $6 \mathrm{~B}$ isolates [7]. Based on the differences in their capsular gene sequences, serotype " $6 \mathrm{E}$ " was assigned to this group in a subsequent study [8]. In a German epidemiological survey, two serogroup 6 isolates could not be assigned to a serotype using factor sera [9]. The latest report by Oliver et al. [10] showed that the two isolates were assigned to new serotypes, "6F" and "6G". 
That study showed that a single codon change in the cps gene, encoding a glycosyl transferase, altered the substrate specificity, caused the biosynthesis of heterogeneous capsule repeating units, and resulted in a new hybrid capsule type. Antigenic formulae and specific factor antisera against serotypes "6E", "6F", and "6G" have not yet been developed, but are necessary for their traditional serotype classification [11]. Because the new serotypes have not been confirmed with the traditional Quellung test, they are designated with quotation marks in the present text.

We did not want to investigate the effects of the vaccine PCV7, because its immunization coverage is too low $(<1 \%)$ to reflect its effectiveness. We set out to identify any new serotypes (serotypes "6E", "6F", and "6G") in serogroup $6 S$. pneumoniae in China and to report the population biology of this group. In our previous study [12], we examined the genetic structures of only serotypes $6 \mathrm{C}$ and $6 \mathrm{D}$, without the most frequent serotypes $6 \mathrm{~A}$ and $6 \mathrm{~B}$. In the present study, we report the STs of all the serogroup 6 isolates and an association between the STs and antibiotic resistance. We intended to use identical isolates in these two studies, but some isolates from our previous study were dead when we went to include them in the present study. Therefore, the present study includes some new isolates collected in 2008-2011 from Shenzhen and Beijing. There were differences in the antibiotic resistance in different clones. For completeness, some published data or similar information from our previous paper have been included in the present results, but these are not included in the analysis or discussion.

\section{Methods}

\section{Pneumococcal isolates}

A total of 225 serogroup 6 S. pneumoniae strains isolated from children in 1997-2011 were included in the study. The details of these $S$. pneumoniae collections have been published previously [13-18]. The isolates were cultured from nasopharyngeal specimens, except those collected from Beijing in 2006-2008 and from Shenzhen in 20092011, which were cultured from hypopharyngeal aspirate specimens. All isolates were identified as serotypes $6 \mathrm{~A}$, 6B, 6C, and 6D with a capsule Quellung test using a pneumotest kit and specific factor antisera (Statens Serum Institut, Copenhagen, Denmark). Typing was performed with phase-contrast microscopy, according to previously reported procedures [19].

A parent and/or legal guardian of each participant signed a written informed consent document before enrollment and before any study procedure was performed. This study was viewed and approved by the Ethics Committee of Beijing Children's Hospital Affiliated to Capital Medical University. No ethical problems existed in this study.

\section{PCR identification of $6 \mathrm{~A}, 6 \mathrm{~B}-\mathrm{I}, 6 \mathrm{~B}-\mathrm{II}, 6 \mathrm{C}, 6 \mathrm{D}$, "6E", "6F", and "6G"}

Chromosomal DNA was extracted from overnight cultures of $S$. pneumoniae isolates grown on 5\% trypticase soy agar (Oxoid Ltd, Basingstoke, England) using the SiMax $^{\mathrm{TM}}$ Genomic DNA Extraction Kit (SBS Genetech Co., Ltd), according to the manufacturer's instructions. The $6 \mathrm{C}$ isolates were differentiated from the $6 \mathrm{~A}$ and "6E" isolates using PCR with primers 5106 and 3101, as described previously $[6,7,20]$, which generated a 1.8 kilobase-pair (kbp) amplicon for $6 \mathrm{C}$ and a $2.0-\mathrm{kbp}$ or $2.3-\mathrm{kbp}$ amplicon for $6 \mathrm{~A}$ or " $6 \mathrm{E}$ ", respectively. This pair of primers was also used to differentiate the $6 \mathrm{D}$ cps locus from that of $6 \mathrm{~B}$, generating a 1.8 -kbp amplicon for $6 \mathrm{D}$ and a $2.0-\mathrm{kbp}$ or $2.3-\mathrm{kbp}$ amplicon for subtypes $6 \mathrm{~B}-\mathrm{I}$ or 6B-II, respectively. The primers 5101 and 3101 used to differentiate $6 \mathrm{~A}$ and "6E", as well as $6 \mathrm{~B}-\mathrm{I}$ and $6 \mathrm{~B}-\mathrm{II}$, more definitively generated a 956-bp amplicon for 6A/ 6B-I and a 1.2-kbp amplicon for "6E"/6B-II [6,7]. The PCR procedures were as previously described [20]. All serotype $6 \mathrm{~A}$ and $6 \mathrm{~B}$ isolates were retested against the factor antiserum $6 \mathrm{~d}$ and all serotype $6 \mathrm{C}$ and $6 \mathrm{D}$ isolates were retested against factor $6 b$ or $6 c$ to identify serotype "6 F" or "6G", respectively, which could not be assigned to serotype $6 \mathrm{~A}$ or $6 \mathrm{C}$ and $6 \mathrm{~B}$ or $6 \mathrm{D}$, respectively. All isolates that reacted with factor $6 \mathrm{~d}$ were subjected to PCR amplification for wciP locus with primers 5106/3101 and wciN locus with primers 5108/3107, as reported previously, to identify serotypes "6 F" and "6G", respectively [21].

\section{Antimicrobial susceptibility}

The minimum inhibitory concentrations (MICs) of all isolates were determined for penicillin, amoxicillin-clavulanic acid, ceftriaxone, cefuroxime, erythromycin, imipenem, levofloxacin, and vancomycin using E-test strips (AB Biodisk, Solna, Sweden), and their susceptibility to tetracycline, sulfamethoxazole-trimethoprim, and chloramphenicol was assessed with disc diffusion tests (Oxoid). The use of the penicillin parenteral (nonmeningitis) breakpoints for susceptible, intermediate, and resistant rates to compare penicillin susceptibilities in this study blurred the differences between the serotypes [22]. Therefore, the oral penicillin V breakpoints were also used to analyze penicillin susceptibility. Streptococcus pneumoniae ATCC49619 was used as the reference strain in the susceptibility tests. Isolates were considered multidrug resistant if they were not susceptible to three or more classes of antimicrobials.

\section{Molecular epidemiology of serogroup 6 strains}

All strains were characterized with multilocus sequence typing (MLST). Bacterial chromosomal DNA was extracted as described above. The PCR fragments of the seven MLST genes (aroE, gdh, gki, recP, spi, xpt, and $d d l$ ) were amplified from the chromosomal DNA. The products 
were sent to BGI Company (Beijing, China) for sequencing on both strands. The STs were determined by comparing the allelic profiles with the recognized STs at the MLST website (http://spneumoniae.mlst.net). The new alleles and allelic profiles identified in the present study have been submitted to the MLST database for name assignment. The eBURST v3 software (available at http://www.mlst.net) was used to estimate the relationships among the isolates and to assign strains to a clonal complex (CC) using the stringent group definition of six of seven shared alleles [23].

\section{Statistical analysis}

The serotyping and antimicrobial resistance data were analyzed with the WHONET 5.3 software, as recommended by the World Health Organization (http://www. who.int/drugresistance/whonetsoftware/en/). The $\chi^{2}$ test, performed with the SPSS software v. 13.0 (SPSS Inc. USA), was used for statistical comparisons. A two-tailed cut-off of $\mathrm{P}<0.05$ was deemed to indicate statistical significance.

\section{Results}

\section{Serotype/subtype distributions and antibiotic susceptibility}

Among the 225 isolates identified as serogroup 6 S. pneumoniae, the rates of serotypes and subtypes 6A, 6B-I, 6B-II, 6C, and 6D were 46.7\% (105/225), 19.6\% (44/225), $25.8 \%$ (58/225), $6.2 \%$ (14/225), and $1.8 \%$ (4/225), respectively. PCR analysis of the serotype 6A isolates with primers $5106 / 3101$ or $5101 / 3101$ always generated the same amplicons as the PCR analysis of 6B-I, indicating that no serotype " $6 \mathrm{E}$ " was present in the serotype $6 \mathrm{~A}$ isolates. No isolate was assigned ambiguously to $6 \mathrm{~A}$ or $6 \mathrm{C}$ or to $6 \mathrm{~B}$ or $6 \mathrm{D}$. The 14 serotype $6 \mathrm{C}$ isolates had identical $w c i P$ and $w c i N$ sequences. The four serotype 6D isolates also had identical wciP and wciN sequences. The length of $w c i P$ was the same as that of the reference sequence CR931638, with 10 random mutations. All wciN sequences were $1125 \mathrm{bp}$ long and were identical to the published 6C sequence (JF911510), which is longer than that of CR931638 (945 bp). No wciN sequence in the present study suggested serotype " $6 \mathrm{~F}$ " or " $6 \mathrm{G}$ ".

The analysis of antibiotic resistance revealed similar results to those in our previous report [12], which are shown in the tables with no further description.

\section{MLST}

The MLST analysis revealed 58 STs, the most common of which were ST982 (52 isolates, 23.1\%), ST90 (33, 14.7\%), ST4542 (17, 7.6\%), and ST2912 (11, 4.9\%). In this study, we identified eight new STs, ST8617-ST8623 and ST8742, and a novel $g k i$ locus ( $g k i 383$ in ST8742). An eBURST analysis identified eight CCs and 26 singletons (Figure 1). Two of the common STs, ST982 and ST2912, were singletons. Six CCs each included 10 or more isolates: CC90 (41 isolates), CC4542 (25 isolates), CC4536 (15 isolates), CC2754 (15 isolates), CC386 (10 isolates), and CC8616 (10 isolates). ST982, ST2912, and these six CCs accounted for $79.6 \%$ of all the isolates tested.

The distribution of the CCs/STs of the serogroup $6 \mathrm{~S}$. pneumoniae isolates according to serotype/subtype is shown in Table 1 and Figure 1. Each serotype/subtype had a main CC/ST that included the majority of the isolates in the serotype/subtype. For instance, the proportion of ST982 in the serotype 6A isolates (44.8\%) was

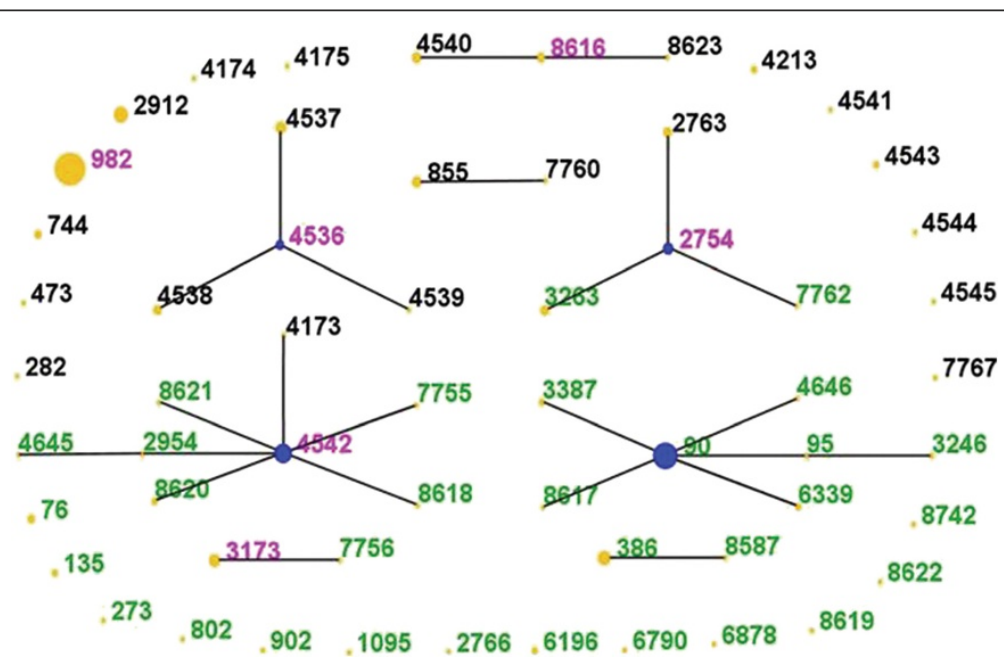

Figure 1 Population snapshot of 225 S. pneumoniae strains revealed by an eBURST analysis. Lines indicate the presence of single-locus-variant links in particular sequence types, indicated by circles. The size of the circle corresponds to the number of isolates belonging to the sequence type (ST). The green ST numbers indicate serotype 6A or 6C isolates, the black numbers indicate 6B or 6D isolates, and the pink numbers indicate isolates from the two groups. 
Table 1 Distributions of CCs/STs of serogroup 6 S. pneumoniae by serotype/subtype

\begin{tabular}{lllllllllll}
\hline Types & No. & ST982 & CC90 & CC4542 & CC4536 & CC2754 & ST2912 & CC8616 & CC386 & Others \\
\hline 6A & 105 & $47(44.8 \%)$ & 0 & $3(2.9 \%)$ & $14(13.3 \%)$ & $6(5.7 \%)$ & $2(1.9 \%)$ & $9(8.6 \%)$ & 0 & $24(22.9 \%)$ \\
6B-I & 44 & 0 & 0 & $20(46.5 \%)$ & $1(2.3 \%)$ & $9(20.9 \%)$ & 0 & $1(2.3 \%)$ & 0 & $12(27.9 \%)$ \\
6B-II & 58 & 0 & $41(69.5 \%)$ & $1(1.7 \%)$ & 0 & 0 & 0 & 0 & $10(16.9 \%)$ & $7(11.9 \%)$ \\
6C & 14 & $1(7.1 \%)$ & 0 & $1(7.1 \%)$ & 0 & 0 & $9(64.3 \%)$ & 0 & 0 & $3(21.4 \%)$ \\
6D & 4 & $4(100 \%)$ & 0 & 0 & 0 & 0 & 0 & 0 & 0 & 0 \\
Total & 225 & $52(23.1 \%)$ & $41(18.2 \%)$ & $25(11.1 \%)$ & $15(6.7 \%)$ & $15(6.7 \%)$ & $11(4.9 \%)$ & $10(4.4 \%)$ & $10(4.4 \%)$ & $46(20.4 \%)$ \\
\hline
\end{tabular}

Note: \%, number of serotypes/subtypes divided by number of CCs/STs.

much higher than that of any other CC/ST $(\leq 13.3 \%)$. Some CCs/STs included several isolates belonging to different serotypes. Most of the ST982 isolates were identified as serotype $6 \mathrm{~A}(47 / 52)$; and the other five isolates were serotypes $6 \mathrm{C}(1 / 52)$ and $6 \mathrm{D}(4 / 52)$. Thus, all four 6D isolates were ST982. Serotypes $6 \mathrm{~A}$ and $6 \mathrm{~B}$ were both detected in the following STs: ST4542 (6A: 3/17; 6B: 14/17), ST2754 (6A: 2/6, 6B: 4/6), ST3173 (6A: 6/7, 6B: 1/7), ST4536 (6A: 3/4, 6B: 1/4), and ST8616 (6A: 3/4, 6B: 1/4). Other STs could only be detected in one serotype.

The CC/ST distributions of the serogroup $6 \mathrm{~S}$. pneumoniae changed significantly during the study period. The common CCs/STs, such as ST982, CC90, CC4542, and CC4536, mainly occurred in 1997-2005, but these isolates decreased or even disappeared in later years. Two CCs among the common CCs/STs, CC2754 and CC386, were mainly found in 2006-2010, with some fluctuations. No CC or ST showed a clearly increasing trend, except the group involving the infrequent "other" CCs/STs ("others" in Table 1). Considerable isolates identified in this study belonged to the infrequent "other" CCs/STs (46/225), especially in 2009-2011 (10/26).

\section{Associations between STs/CCs and antimicrobial resistance}

The numbers and percentages of isolates not susceptible to oral penicillin and other antibiotics are summarized according to CCs/STs in Tables 2 and 3. Notably, all
CC90 isolates were not susceptible to oral penicillin, with high $\mathrm{MIC}_{50}$ and $\mathrm{MIC}_{90}$ values, whereas other common CCs/STs were usually susceptible, except that some intermediately susceptible strains (40.0\%) were present in CC386. More than half the infrequent "other" CC/ST isolates were not susceptible to oral penicillin. High rates of nonsusceptibility to cefuroxime were found in CC90 (92.7\%) and the infrequent "other" CCs/STs (45.6\%). The differences in some other antimicrobial resistances between the CCs/STs are shown in Table 3.

The distributions of serotypes/subtypes and CCs/STs in the oral-penicillin-nonsusceptible strains (OPNSP) were analyzed further, as shown in Table 4. Although $6 \mathrm{~B}-\mathrm{II}$ was the main subtype in the total OPNSP strains, its proportion decreased from $82.1 \%$ in 2000-2002 to $41.7 \%$ in $2009-2011\left(\chi^{2}=5.6, P<0.05\right)$. The proportion of serotype $6 \mathrm{~A}$ strains increased significantly from $10.3 \%$ to $50.0 \%$ during the same period $\left(\chi^{2}=6.8, P<0.01\right)$. The constitution of CCs/STs among the OPNSP also changed. The proportions of CC90 and CC386 that were identified as subtype 6B-II decreased. However, the proportions of CC855 (including six serotype 6A isolates) increased, as did the proportions of CC3173 (including six serotype $6 \mathrm{~A}$ isolates and two subtype 6B-I isolates). The distribution of penicillin MICs between the CCs/STs did not differ, except that the MICs of the CC386 isolates reached $0.125 \mu \mathrm{g} / \mathrm{ml}$.

Table 2 Penicillin susceptibility in serogroup 6 S. pneumoniae by CCs/STs

\begin{tabular}{|c|c|c|c|c|c|c|}
\hline \multirow[t]{2}{*}{ CCs/STs } & \multirow[t]{2}{*}{ No. isolates } & \multicolumn{2}{|c|}{ Oral penicillin breakpoints } & \multirow[t]{2}{*}{ MIC50 $(\mu \mathrm{g} / \mathrm{mL})$} & \multirow[t]{2}{*}{ MIC90 $(\mu \mathrm{g} / \mathrm{mL})$} & \multirow[t]{2}{*}{ MIC range $(\mu \mathrm{g} / \mathrm{mL})$} \\
\hline & & $\mathrm{I}$ & $\mathbf{R}$ & & & \\
\hline ST982 & 52 & 0 & 0 & 0.032 & 0.049 & $0.001-0.064$ \\
\hline CC90 & 41 & $36(87.8 \%)$ & $5(12.2 \%)$ & 1 & 1.5 & $0.25-2$ \\
\hline CC4542 & 25 & 0 & 0 & 0.016 & 0.023 & $0.003-0.032$ \\
\hline ST2912 & 11 & 0 & 0 & 0.032 & 0.049 & $0.016-0.049$ \\
\hline CC4536 & 15 & 0 & 0 & 0.016 & 0.032 & $0.016-0.064$ \\
\hline CC2754 & 11 & 0 & 0 & 0.023 & 0.032 & $0.008-0.064$ \\
\hline CC8616 & 10 & 0 & 0 & 0.016 & 0.023 & $0.002-0.023$ \\
\hline CC386 & 10 & $4(40.0 \%)$ & 0 & 0.064 & 0.125 & $0.032-0.125$ \\
\hline Others & 46 & $22(47.8 \%)$ & $4(8.7 \%)$ & 0.19 & 0.75 & $0.008-2$ \\
\hline Total & 225 & $63(28.0 \%)$ & $9(4 \%)$ & 0.032 & 1 & $0.001-2$ \\
\hline
\end{tabular}


Table 3 Resistance of serogroup 6S. pneumoniae to several antimicrobials by CCs/STs

\begin{tabular}{|c|c|c|c|c|c|c|c|c|c|c|c|}
\hline \multirow[t]{2}{*}{ CC/STs } & \multirow[t]{2}{*}{ No. } & \multicolumn{2}{|c|}{$\mathrm{CXM}^{\mathrm{a}}$} & \multirow{2}{*}{$\begin{array}{l}\text { IPM }^{\mathrm{a} *} \\
I \%\end{array}$} & \multirow{2}{*}{$\begin{array}{l}\mathrm{ERY}^{\mathrm{a} *} \\
\mathrm{R} \%\end{array}$} & \multirow{2}{*}{$\begin{array}{l}\mathrm{CHL}^{\mathrm{a} *} \\
\mathrm{R} \%\end{array}$} & \multicolumn{2}{|c|}{$\mathrm{TCY}^{\mathrm{a}}$} & \multicolumn{2}{|l|}{$\mathrm{SXT}^{\mathrm{a}}$} & \multirow{2}{*}{$\begin{array}{l}M^{M R^{2}} \\
(\%)\end{array}$} \\
\hline & & $1 \%$ & $\mathrm{R} \%$ & & & & $1 \%$ & $\mathrm{R} \%$ & $1 \%$ & $\mathrm{R} \%$ & \\
\hline ST982 & 52 & 0 & 0 & 0 & 98.1 & 3.8 & 1.9 & 98.1 & 1.9 & 98.1 & 98.1 \\
\hline CC90 & 41 & 4.9 & 87.8 & 19.5 & 100 & 58.5 & 0 & 95.1 & 4.9 & 92.7 & 100 \\
\hline CC4542 & 25 & 0 & 0 & 8.0 & 96.0 & 8.0 & 0 & 100 & 4.0 & 88.0 & 92.0 \\
\hline ST2912 & 11 & 0 & 0 & 0 & 100 & 0 & 36.4 & 45.5 & 9.1 & 63.6 & 63.6 \\
\hline CC4536 & 15 & 6.7 & 0 & 0 & 66.7 & 46.7 & 0 & 100 & 20.0 & 46.7 & 73.3 \\
\hline CC2754 & 15 & 0 & 6.7 & 0 & 100 & 6.7 & 0 & 93.3 & 13.3 & 86.7 & 93.3 \\
\hline CC8616 & 10 & 0 & 0 & 10.0 & 90.0 & 10.0 & 0 & 90.0 & 0 & 90.0 & 90.0 \\
\hline CC386 & 10 & 0 & 0 & 0 & 100 & 0 & 10.0 & 80.0 & 0 & 10.0 & 40.0 \\
\hline Others & 46 & 6.5 & 39.1 & 8.7 & 93.5 & 26.1 & 4.3 & 80.4 & 15.2 & 63.0 & 91.3 \\
\hline Total & 225 & 2.7 & 24.4 & 6.7 & 95.1 & 21.8 & 3.6 & 90.2 & 7.6 & 78.7 & 89.8 \\
\hline
\end{tabular}

Note: All 225 strains were susceptible to amoxicillin-clavulanic acid, ceftriaxone, vancomycin, and levofloxacin.

${ }^{a}$ CXM: cefuroxime; IPM: imipenem; ERY: erythromycin; CHL: chloramphenicol; TCY: tetracycline; SXT: sulfamethoxazole; MDR: multidrug resistance.

${ }^{*}$ No intermediate or resistant isolate was identified.

\section{Discussion}

Recently, Ko et al. [8] identified a distinct group among serotypes 6A and 6B S. pneumoniae isolates, designated serotype " $6 \mathrm{E}$ ". Based on sequence data, the serotype " $6 \mathrm{E}$ " isolates from traditional serotype $6 \mathrm{~B}$ were the same as those from subtype 6B-II in the present study, which is exactly consistent with subtype 6B-III reported by Elberst et al. [24]. Subtype 6B-II was isolated frequently in the present study. However, no serotype " $6 \mathrm{E}$ " isolate was identified in traditional serotype 6A with PCR.

As-yet-unconfirmed serotype " $6 \mathrm{E}$ " has only been identified based on its capsular gene sequence [8] because no new serotype including both traditional serotypes $6 \mathrm{~A}$ and $6 \mathrm{~B}$ can be constructed within the serological typing system [11]. If differences in gene sequences indicate new serotypes, two new serotypes must be individually recognized within the traditional serotypes $6 \mathrm{~A}$ and $6 \mathrm{~B}$.

All the common CCs/STs included more than one serotype or subtype, except CC90 and CC386, which included subtype 6B-II isolates only. One subtype 6B-II isolate belonged to $\mathrm{CC} 4542$, which otherwise included only serotype $6 \mathrm{~A}$ and subtype $6 \mathrm{~B}-\mathrm{I}$ isolates. However, this isolate had a unique ST, ST8618. Two classes of $c p s$ sequences have been reported for serogroup 6 isolates.
The class 2 cps sequences are limited to a few serotype $6 \mathrm{~B}$ isolates [25], which are in fact subtype 6B-II. The present data show that our subtype 6B-II isolates displayed genetic backgrounds different from those of other serotypes/subtypes.

Our previous studies have suggested that a clonal shift occurred in serotype 19F and 23F isolates between 1997 and 2010. In the serotype 19F S. pneumoniae isolates, the spread of CC271 with high $\beta$-lactam-antibiotic resistance may be driven by the selective pressure applied with the overuse of antibiotics, whereas the spread of susceptible CC983 is limited. CC271 became the main population of serotype 19F S. pneumoniae after 2003 [26]. In the serotype 23F S. pneumoniae isolates, ST81 (with high $\beta$-lactam-antibiotic resistance) took the place of ST342 and became the absolutely predominant population in 2010 [27]. Both CC271 and ST81 are also important international epidemic clones [26,27]. We noted that selection by antibiotic abuse caused an increase in subtype 6B-II isolates with significantly higher $\beta$-lactamantibiotic resistance than that of other serogroup $6 \mathrm{~S}$. pneumoniae isolates in 1997-2008 [12]. The present data show that most subtype 6B-II isolates belong to CC90. ST90 is one of the globally epidemic resistant

Table 4 Distributions of serotypes/subtypes in oral-penicillin-nonsusceptible isolates in China in 2000-2011

\begin{tabular}{|c|c|c|c|c|c|c|c|c|c|}
\hline \multirow[t]{2}{*}{ Years } & \multirow[t]{2}{*}{ No. } & \multicolumn{3}{|c|}{ Serotypes/subtypes } & \multicolumn{5}{|l|}{ CCs/STs } \\
\hline & & $6 \mathrm{~A}$ & 6B-I & 6B-II & CC90 & CC386 & CC855 & CC3173 & Singletons* \\
\hline 2000-2002 & 39 & $4(10.3 \%)$ & $3(7.7 \%)$ & $32(82.1 \%)$ & $27(69.2 \%)$ & $3(7.7 \%)$ & $2(5.1 \%)$ & $0(0.0 \%)$ & ST76 (3), ST473,ST802, ST902, ST4545 \\
\hline 2003-2005 & 9 & $2(22.2 \%)$ & $0(0.0 \%)$ & $7(77.8 \%)$ & $6(66.7 \%)$ & $0(0.0 \%)$ & $0(0.0 \%)$ & $1(11.1 \%)$ & ST282,ST8742 \\
\hline 2006-2008 & 12 & $3(25.0 \%)$ & $2(16.7 \%)$ & $7(58.3 \%)$ & $6(50.0 \%)$ & $0(0.0 \%)$ & $2(16.7)$ & $2(16.7 \%)$ & ST135 \\
\hline 2009-2011 & 12 & $6(50.0 \%)$ & $1(8.3 \%)$ & $5(41.7 \%)$ & $2(16.7 \%)$ & $1(8.3 \%)$ & $2(16.7 \%)$ & $5(41.7 \%)$ & ST135,ST2766 \\
\hline Total & 71 & $15(21.1 \%)$ & $6(8.5 \%)$ & $51(71.8 \%)$ & $41(57.7 \%)$ & $4(5.6 \%)$ & $6(8.5 \%)$ & $8(11.3 \%)$ & \\
\hline
\end{tabular}

Note: No oral-penicillin-nonsusceptible strain was present in 1997-1999.

*The number of isolates is given in parentheses if more than one isolate was identified. 
clones, Spain ${ }^{6 \mathrm{~B}}-2$ (http://web1.sph.emory.edu/PMEN/index. $\mathrm{html})$. CC90 isolates were the main components of the OPNSP in our study. However, isolates belonging to this clone decreased in 2009-2011, and its substitutes, CC855 and CC3173, did not show higher $\beta$-lactam-antibiotic resistance. Therefore, this replacement cannot be explained by antibiotic overuse and remains undefined at present. Most of the CC855 and CC3173 isolates were identified as serotype $6 \mathrm{~A}$, which is not covered by the 7 -valent pneumococcal conjugated vaccine (PCV7). The proportion of serotype 6A isolates in the OPNSP increased significantly (Table 4). This replacement may be attributable to immunization with PCV7, which was used after September 2008 in China. However, this rationale is not convincing because the vaccine immunization coverage was so low (less than 1\%) that the spread of serotypes 19F and 23F was not restricted at all $[26,27]$.

The $\mathrm{MIC}_{90}$ and maximum MIC of penicillin in serogroup 6S. pneumoniae isolates remained at $1 \mu \mathrm{g} / \mathrm{ml}$ and $2 \mu \mathrm{g} / \mathrm{ml}$, respectively, from 2000 to 2010 in China (Table 2), whereas the $\mathrm{MIC}_{90}$ of serotypes $19 \mathrm{~F}$ and $23 \mathrm{~F}$ increased to $2 \mu \mathrm{g} / \mathrm{ml}$ and the maximum value was $8 \mu \mathrm{g} / \mathrm{ml}$, and even reached $256 \mu \mathrm{g} / \mathrm{ml}[28,29]$. The present data also show the absence of salient resistant clones in serogroup $6 S$. pneumoniae. They all may be under antibiotic selective pressure. Therefore, the CC/ST components of this group have not changed regularly, like serotypes $19 \mathrm{~F}$ and $23 \mathrm{~F}$. This speculation corresponds to the decreasing frequency of serogroup 6 among all S. pneumoniae isolates over time [12]. The population biology of this serogroup should be monitored continuously in the future.

\section{Conclusions}

In summary, this study clarifies the serotypes/subtypes and CCs/STs of group 6 S. pneumoniae present in China. Serotype "6E" was not identified in the traditional 6A isolates, and neither serotype " 6 F" nor " $6 G$ " was assigned in our collection. Although the subtype 6B-II isolates were the main constituents of the OPNSP, their place was taken by serotype $6 \mathrm{~A}$ isolates over time. The genetic constitution of the group 6 S. pneumoniae and its changes showed distinct regional characters. Unlike the serotype $19 \mathrm{~F}$ and $23 \mathrm{~F}$ isolates, no salient resistant clones were identified in serogroup $6 S$. pneumoniae. Further long-term surveys of serogroup $6 S$. pneumoniae are required to monitor the clonal prevalence and antimicrobial resistance in this important human pathogen.

\section{Abbreviations}

PCR: Polymerase chain reaction; ST: Sequence type; MLST: Multilocus sequence typing; CC: Clonal complex; MDR: Multidrug resistant; MIC: Minimum inhibitory concentration.

\section{Competing interests}

The authors declare that they have no competing interests.

\section{Authors' contributions}

WS, KY, MH, SY, and YY designed the study. WS and SY performed the antimicrobial susceptibility tests. MH and KY performed the molecular epidemiological PCR. WS, KY, and YY collected the data, analyzed them, interpreted the results, and drafted the manuscript. All authors reviewed and revised the manuscript and approved the final version.

\section{Acknowledgments}

We sincerely thank the staff of the following esteemed institutions for providing isolates during the several collection programs: Shenzhen Children's Hospital (Yue-jie Zheng, Rui-zhen Zhao), Pediatric Hospital of Fudan University (Li-bo Wang, Chuan-qing Wang), Shengjing Hospital Affiliated to China Medical University (Yun-xiao Shang, Yong Liu), Nanjing Children's Hospital (De-yu Zhao, Hui-yun Wang), Tianjin Children's Hospital (Li-ya Wan), Chongqing Children's Hospital Affiliated to Chongqing Medical University (Lan Liu), Yuying Children's Hospital of Wenzhou Medical College (Changchong Li), Suzhou University Pediatric Hospital (Wei Ji, Yun-fang Ding), Anhui Medical University Affiliated Hospital (Ya-ting Wang, Yuan-hong Xu), First Affiliated Hospital of Xinjiang Medical University (Pei-ru Xu, Ping Ji), Pediatric Hospital of Shanghai Jiao Tong University (Quan Lu, Hong Zhang), Guangzhou Children's Hospital (Li Deng, Qiu-lian Deng), and Xijing Hospital of the Fourth Military Medical University (Guo-cheng Zhang). We are grateful for the use of the MLST database, which is located at Imperial College London and funded by the Wellcome Trust.

Received: 30 April 2014 Accepted: 19 August 2014

Published: 27 August 2014

\section{References}

1. World Health Organization: Pneumococcal vaccines WHO position paper. Wkly Epidemiol Rec 2012, 87:129-144.

2. Park IH, Pritchard DG, Cartee R, Brandao A, Brandileone MC, Nahm MH: Discovery of a new capsular serotype (6C) within serogroup 6 of Streptococcus pneumoniae. J Clin Microbiol 2007, 45:1225-1233.

3. Jin P, Kong F, Xiao M, Oftadeh S, Zhou F, Liu C, Russell F, Gilbert GL: First report of putative Streptococcus pneumoniae serotype 6D among nasopharyngeal isolates from Fijian children. J Infect Dis 2009, 200:1375-1380.

4. Lambertsen L, Kerrn MB: Test of a novel Streptococcus pneumoniae serotype 6 C type specific polyclonal antiserum (factor antiserum $6 \mathrm{~d}$ ) and characterization of serotype 6C isolates in Denmark. BMC Infect Dis 2010, 10:282.

5. Yao KH, Liu ZJ, Yang YH: How many factor antisera can be found for serogroup 6 Streptococcus pneumoniae? J Clin Microbiol 2010, 48:3046.

6. Jin P, Xiao M, Kong F, Oftadeh S, Zhou F, Liu C, Gilbert GL: Simple, accurate, serotype-specific PCR assay to differentiate Streptococcus pneumoniae serotypes 6A, 6B, and 6C. J Clin Microbiol 2009, 47:2470-2474.

7. Song JH, Baek JY, Ko KS: Comparison of capsular genes of Streptococcus pneumoniae serotype 6A, 6B, 6C, and 6D isolates. J Clin Microbiol 2011, 49:1758-1764

8. Ko KS, Baek JY, Song JH: Capsular gene sequences and genotypes of 'serotype 6E' Streptococcus pneumoniae isolates. J Clin Microbio/ 2013, 51:3395-3399.

9. van der Linden M, Winkel N, Küntzel S, Farkas A, Perniciaro SR, Imohl M: Epidemiology of Streptococcus pneumoniae serogroup 6 isolates from IPD in children and adults in Germany. PLoS One 2013, 8:e60848.

10. Oliver MB, van der Linden MP, Küntzel SA, Saad JS, Nahm MH: Discovery of Streptococcus pneumoniae serotype 6 variants with glycosyltransferases synthesizing two differing repeating units. J Biol Chem 2013, 288:25976-25985.

11. Henrichsen J: Six newly recognized types of Streptococcus pneumoniae. J Clin Microbiol 1995, 33:2759-2762.

12. Yao KH, Liu ZJ, Yu JG, Yu SJ, Yuan L, Nahm MH, Yang YH: Type distribution of serogroup 6 Streptococcus pneumoniae and molecular epidemiology of newly identified serotypes 6C and 6D in China. Diag Microbil Infect Dis 2011, 70:291-298.

13. Li J, Yang YH, Yu SJ, Yuan L, Hu YY: Study on resistance to antibiotics to Streptococcus pneumoniae. Zhonghua Erke Zazhi 2011, 37:408-411 (in Chinese).

14. Xue L, Yao K, Xie G, Zheng Y, Wang C, Shang Y, Wang H, Wan L, Liu L, Li C, Ji W, Xu X, Wang Y, Xu P, Liu Z, Yu S, Yang Y: Serotype distribution and antimicrobial resistance of Streptococcus pneumoniae isolates that cause invasive disease among Chinese children. Clin Infect Dis 2010, 50:741-744. 
15. Yao K, Shen X, Yu S, Lu Q, Deng L, Ye Q, Zhang H, Deng Q, Hu Y, Yang Y: Antimicrobial resistance and serotypes of nasopharyngeal strains of Streptococcus pneumoniae in Chinese children with acute respiratory infections. J Int Med Res 2007, 35:253-267.

16. Yao KH, Wang LB, Zhao GM, Zheng YJ, Deng L: Study on antimicrobial resistance in different serotypes of Streptococcus pneumoniae isolated from pediatric patients hospitalized for pneumonia. Linchuang Erke Zazhi 2009, 27:524-527,533 (in Chinese).

17. Yu J, Carvalho Mda G, Beall B, Nahm MH: A rapid pneumococcal serotyping system based on monoclonal antibodies and PCR. J Med Microbiol 2008, 57:171-178.

18. Ma X, Zhao R, Ma Z, Yao K, Yu S, Zheng Y, Yang Y: Serotype distribution and antimicrobial resistance of streptococcus pneumoniae isolates causing invasive diseases from Shenzhen children's hospital. PLoS One 2013, 8:e67507.

19. Sørensen UBS: Typing of pneumococci by using 12 pooled antisera. J Clin Microbiol 1993, 31:2097-2100

20. Carvalho Mda G, Pimenta FC, Gertz RE, Joshi HH, Trujillo AA, Keys LE, Findley J, Moura IS, Park IH, Hollingshead SK, Pilishvili T, Whitney CG, Nahm MH, Beall BW: PCR-based quantitation and clonal diversity of the current prevalent invasive serogroup 6 pneumococcal serotype, 6C, in the United States in 1999 and 2006 to 2007. J Clin Microbiol 2009, 47:554-559.

21. Park IH, Park S, Hollingshead SK, Nahm MH: Genetic basis for the new pneumococcal serotype, 6C. Infect Immun 2007, 75:4482-4489.

22. Clinical Laboratory Standards Institute (CLSI): Performance Standards for Antimicrobial Susceptibility Testing; Twenty-Second Informational Supplement, M100-S22. Wayne, PA: CLSI; 2012.

23. Feil EJ, Li BC, Aanensen DM, Hanage WP, Spratt BG: eBURST: inferring patterns of evolutionary descent among clusters of related bacterial genotypes from multilocus sequence typing data. J Bacterio/ 2004, 186:1518-1530.

24. Elberse K, Witteveen S, van der Heide H, van de Pol I, Schot C, van der Ende I, Berbers $G$, Schouls L: Sequence diversity within the capsular genes of Streptococcus pneumoniae serogroup 6 and 19. PLoS One 2011, 6:e25018.

25. Bratcher PE, Park IH, Oliver MB, Hortal M, Camilli R, Hollingshead SK, Camou T, Nahm MH: Evolution of the capsular gene locus of Streptococcus pneumoniae serogroup 6. Microbiology 2011, 157:189-198.

26. Li QH, Yao KH, Yu SJ, He MM, Shi W: The antibiotic resistance and molecular epidemiology of serotype $19 \mathrm{~F}$ Streptococcus pneumoniae isolated from Beijing children's hospital in China. Zhongguoshiyong Erke Zazhi 2013, 28:179-183 (in Chinese)

27. Ma X, Yao KH, Yu SJ, Zhou L, Li QH, Shi W, He MM, Yang YH: Genotype replacement within serotype $23 \mathrm{~F}$ Streptococcus pneumoniae in Beijing, China: characterization of serotype $23 \mathrm{~F}$. Epidemiol Infect 2013, 141:1690-1696

28. Yao KH, Lu Q, Deng L, Yu SJ, Zhang H: Serotype distribution and resistance to $\beta$-lactams of Streptococcus pneumoniae isolated from children in Beijing, Shanghai and Guangzhou, 2000-2002. Chin J Pediatric 2006, 44:928-932 (in Chinese).

29. Yao KH, Wang LB, Zhao GM, Yue-Jie Zheng YJ, Deng L, Huang JF, Wang JX, Zhao RZ, Deng QL, Hu YH, Yu SJ, Yang YH, Young M: Pneumococcal serotype distribution and antimicrobial resistance in Chinese children hospitalized for pneumonia. Vaccine 2011, 29:2296-2301.

\section{Submit your next manuscript to BioMed Central and take full advantage of:}

- Convenient online submission

- Thorough peer review

- No space constraints or color figure charges

- Immediate publication on acceptance

- Inclusion in PubMed, CAS, Scopus and Google Scholar

- Research which is freely available for redistribution

Submit your manuscript at www.biomedcentral.com/submit
C Biomed Central 